\title{
Elevated carcinoembryonic antigen in patients with COVID-19 pneumonia
}

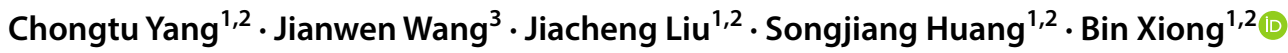

Received: 20 June 2020 / Accepted: 4 August 2020 / Published online: 28 August 2020

c) Springer-Verlag GmbH Germany, part of Springer Nature 2020

\begin{abstract}
Purpose Coronavirus disease 2019 (COVID-19) tends to affect multiple organs and induce abnormal laboratory parameters. We designed this study to investigate the association between carcinoembryonic antigen (CEA) elevation and SARS-CoV-2 infection.

Methods We retrospectively analyzed 177 patients with confirmed SARS-CoV-2 infection who received plasma CEA assays during hospitalization. Patients with other causes of CEA elevation were excluded. Data regarding epidemiological and demographical characteristics, clinical symptoms, laboratory tests, and outcomes were analyzed. Linear regression analysis was used to evaluate the correlation between CEA levels and inflammation severity.

Results 171 patients were included in the final study and 32 patients (18.7\%) had raised serum of CEA ( $>5 \mathrm{ng} / \mathrm{ml})$, with a median (range) age of 66 (53-86). The median [interquartile range (IQR)] CEA level was $11.4 \mathrm{ng} / \mathrm{ml}(8.1-21.6)$, which was significantly higher than the upper limit of reference range. CEA level between $5-10 \mathrm{ng} / \mathrm{ml}$ was in 11 patients, $10-15 \mathrm{ng} /$ $\mathrm{ml}$ in 10 patients, and $>15 \mathrm{ng} / \mathrm{ml}$ in 11 patients. No correlation was found between CEA levels and lymphocyte $\left(R^{2}=0.055\right.$; $P=0.10)$ nor CRP $\left(R^{2}=0.026 ; P=0.38\right)$. The median levels of CEA were $20.0 \mathrm{ng} / \mathrm{ml}(\mathrm{IQR}, 14.7-23.0)$ in non-survivors and $10.9 \mathrm{ng} / \mathrm{ml}$ (IQR 7.5-16.1) in survivors, and the difference between two groups was statistically significant $(P=0.048)$. Conclusion SARS-CoV-2 infection might be another cause of CEA elevation, with nearly $20 \%$ of patients experienced transient and marked CEA increment during COVID-19 pneumonia. The false-positive results of CEA elevation might have clinical significance for patients with colorectal cancer.
\end{abstract}

Keywords Carcinoembryonic antigen · Coronavirus disease 2019 · SARS-CoV-2 · Gastrointestinal damage

\section{Bin Xiong}

herr_xiong@126.com

Chongtu Yang

henrys1011@163.com

Jianwen Wang

blissmark@sina.com

Jiacheng Liu

jiacheng6jc@163.com

Songjiang Huang

hsjhzkjdx@163.com

1 Department of Radiology, Union Hospital, Tongji Medical College, Huazhong University of Science and Technology, Jiefang Avenue \#1277, Wuhan 430022, China

2 Hubei Province Key Laboratory of Molecular Imaging, Wuhan, China

3 Tuberculosis and Respiratory Department, Wuhan Jinyintan Hospital, Wuhan, China
Coronavirus disease 2019 (COVID-19) caused by SARS$\mathrm{CoV}-2$ infection has now become an international public health crisis. Mounting evidence suggests that this novel disease can affect multiple organs despite lung, such as heart, liver and gastrointestinal tract, and results in abnormalities of several biomarkers (Lin et al. 2020; Guan et al. 2019). Carcinoembryonic antigen (CEA) is a glycoprotein formed in colonic epithelium during embryonic period, which has been widely utilized as a tumor marker to monitor tumor progression (Goldenberg et al. 1981). We found a portion of patients with COVID-19 pneumonia had raised serum of CEA, and we designed this study to investigate the association between CEA elevation and SARS-CoV-2 infection.

We retrospectively analyzed 177 consecutive patients with laboratory confirmed COVID-19 who admitted to Wuhan Jinyintan Hospital between January 1 and February 28,2020 . At the early stage of this epidemic, all patients admitted to our center received CEA tests as well as other 
non-routine examinations. Patients with other potential causes of CEA elevation like recent smoking history, malignant tumors, or non-malignant conditions (such as inflammatory bowel disease or pancreatitis) were excluded. Nonparametric continuous variables were expressed as median (interquartile range) and compared using Mann-Whitney $U$ test. Qualitative variables were expressed as number (percentage) and compared by means of Chi-squared or twotailed Fisher's exact test. Linear regression analysis was used to evaluate the correlation between CEA levels and inflammation severity [judged by lymphocyte count and C-reactive protein (CRP)]. A two-sided $\alpha$ of less than 0.05 was considered statistically significant. All statistical analyses were processed using IBM SPSS (version 25.0). The study protocol was approved by the institutional ethics committee of Wuhan Union Hospital and Wuhan Jinyintan Hospital. Informed consent was waived given the retrospective nature of this study.

One hundred and seventy-one patients were enrolled in the final cohort, including 23 patients who were admitted to the intensive care unit. Among them, 32 patients (18.7\%) had raised serum of CEA $(>5 \mathrm{ng} / \mathrm{ml})$, with a median age of 66 years (range 53-86). The median [interquartile range (IQR)] CEA level was $11.4 \mathrm{ng} / \mathrm{ml}(8.1-21.6)$, which was significantly higher than the upper limit of reference range. CEA level between $5-10 \mathrm{ng} / \mathrm{ml}$ was in 11 patients, $10-15 \mathrm{ng} /$ $\mathrm{ml}$ in 10 patients, and $>15 \mathrm{ng} / \mathrm{ml}$ in 11 patients. The highest level of CEA was $63 \mathrm{ng} / \mathrm{ml}$. The median time from disease onset to the first detection of CEA was 12 days (IQR 9-16) (Table 1).

Serial determinations of CEA (CEA test $\geq 2$ times) were available in 13 patients. CEA levels returned to normal in three patients, with an interval of 14, 26 and 40 days, respectively, from their disease onset. CEA levels of other ten patients remained abnormal until their last determination, but seven of them showed a decrease, with a median interval of 24 days (range 19-32) from disease onset.

All 32 patients had definite outcomes, of whom 7 died and 25 recovered and discharged from hospital. The median levels of CEA were $20.0 \mathrm{ng} / \mathrm{ml}$ (IQR 14.7-23.0) in non-survivors and $10.9 \mathrm{ng} / \mathrm{ml}$ (IQR 7.5-16.1) in survivors, and the difference between two groups was statistically significant $(P=0.048)$. No correlation was found between CEA levels and lymphocyte $\left(R^{2}=0.055 ; P=0.10\right)$ nor $\mathrm{CRP}\left(R^{2}=0.026\right.$; $P=0.38$ ).

The exact mechanism of CEA elevation during COVID19 pneumonia remains elusive. One possible explanation is that CEA as an acute phase protein, its increment may be associated with inflammation induced by viral infection. However, no correlation was found between CEA level and severity of inflammation in the present study, indicating this assumption may not be reasonable. Another possible mechanism may be related to the gastrointestinal damage caused by viral infection. As previously reported, the regeneration
Table 1 Baseline characteristics of patients with elevated serum of CEA concentrations

\begin{tabular}{|c|c|c|c|c|}
\hline & Total $(n=32)$ & Survivor $(n=25)$ & Non-survivor $(n=7)$ & $P$ value \\
\hline Age- years $^{\mathrm{a}}$ & $66.0(61.0-76.0)$ & $65.0(59.0-71.0)$ & $74.0(63.0-84.0)$ & 0.242 \\
\hline Sex $(\text { male })^{\mathrm{b}}$ & $18(56.2 \%)$ & $11(44.0 \%)$ & $7(100 \%)$ & 0.01 \\
\hline Co-existing disease ${ }^{b}$ & & & & 0.614 \\
\hline COPD & $2(6.2 \%)$ & $1(4 \%)$ & $1(14.2 \%)$ & \\
\hline Hypertension & $17(52.1 \%)$ & $14(56 \%)$ & $3(42.8 \%)$ & \\
\hline Diabetes & $10(31.2 \%)$ & $9(36 \%)$ & $1(14.2 \%)$ & \\
\hline Cerebrovascular disease & $1(3.1 \%)$ & $1(4 \%)$ & $0(0 \%)$ & \\
\hline \multicolumn{5}{|l|}{ Laboratory parameters $^{\mathrm{a}}$} \\
\hline Leucocyte count $(\times 109 / 1)$ & $8.1(6.1-10.4)$ & $7.8(5.9-9.9)$ & $9.3(6.9-13.0)$ & 0.281 \\
\hline Neutrophil count $(\times 109 / 1)$ & $6.6(4.9-9.0)$ & $6.2(4.9-8.6)$ & $7.9(6.1-12.5)$ & 0.148 \\
\hline Lymphocyte count $(\times 109 / 1)$ & $0.87(0.61-1.25)$ & $0.95(0.63-1.5)$ & $0.69(0.35-1.1)$ & 0.175 \\
\hline C-reactive protein $(\mathrm{mg} / \mathrm{l})$ & $59(24-139)$ & $48(18-115)$ & $148(45-160)$ & 0.02 \\
\hline $\mathrm{ESR}(\mathrm{mm} / \mathrm{h})$ & $56(30-71)$ & $67(35-76)$ & $35(7-44)$ & 0.0 \\
\hline Serum CEA levels $(\mathrm{ng} / \mathrm{ml})^{\mathrm{a}}$ & $11.4(8.1-21.6)$ & $10.9(7.5-16.1)$ & $20.0(14.7-23.0)$ & 0.048 \\
\hline \multicolumn{5}{|l|}{ Time from disease onset to ${ }^{a}$} \\
\hline Hospital admission—days & $11(8-15)$ & $10(8-15)$ & $15(12-23)$ & 0.161 \\
\hline First CEA test—days & $12(9-16)$ & $11(8-16)$ & $15(12-24)$ & 0.191 \\
\hline
\end{tabular}

$C O P D$ chronic obstructive pulmonary disease; $C E A$ carcinoembryonic antigen; $E S R$ erythrocyte sedimentation rate

${ }^{a}$ Nonparametric continuous variables were expressed as median (interquartile range) and compared using Mann-Whitney $U$ test

${ }^{\mathrm{b}}$ Qualitative variables were expressed as number (percentage) and compared by means of Chi-squared or two-tailed Fisher's exact test 
and/or degeneration of intestinal mucosa in patients with gastrointestinal disease was similar to the process of embryonic development and carcinoma progression, which was the leading course of CEA increment (Rule et al. 1973). SARS$\mathrm{CoV}-2$, which can identify angiotensin-converting enzyme 2 (ACE2) through its spike protein and consequently enter target cells, has been proved to infect gastrointestinal glandular cells (Hoffmann et al. 2020; Jin et al. 2019; Xiao et al. 2020). These evidences indicate that SARS-CoV-2 infection might contribute to CEA elevation secondary to mucosal damage.

Previous studies showed that elevated CEA was correlated with disease severity (manifested by declined pulmonary function) in patients with idiopathic pulmonary fibrosis (Fahim et al. 2012). Similarly, CEA level was significantly higher in non-survivors in our study, suggesting this biomarker may be predictive of poor prognosis. In addition, considering the clinical and prognostic value of CEA in patients with confirmed colorectal malignancy, as its failure to decrease to normal after surgical resection indicates residual lesion and its rapid and marked increment indicates recurrence or metastasis, regular assays of CEA is widely used as an effective non-invasive method for postoperative surveillance. Our results demonstrated that transient and marked CEA elevation was not rare during the clinical course of COVID-19. Thus, physicians should be aware that a single measurement of CEA in COVID-19 patients may be a false-positive result induced by viral infection and may not be suggestive of tumor progression or remission, and sequential assays should be conducted subsequently. However, as none of our participants had any clinical indication of malignancy, further studies are needed to investigate the role of CEA level in COVID-19 patients accompanied with colorectal cancer.

The present study has several limitations. First, since CEA increment was not frequent in COVID-19 patients and we enrolled patients within a short period, the sample size was relatively small and thus no firm conclusions could be drawn. Second, as a pilot study which focused on reporting an abnormal increment of a biomarker during COVID-19 pneumonia, we did not perform basic research to confirm this hypothesis. Therefore, further studies are needed to investigate the underlying pathophysiological mechanisms.

In conclusion, SARS-CoV-2 infection might cause transient and marked CEA elevation especially in non-survivors, and this abnormal increment was not correlated with the severity of inflammation. Future studies on the mechanisms of CEA elevation and the role of CEA in predicting prognosis are warranted.

Author contributions BX and CT designed the whole study. BX supervised the whole project. CT and JC performed data analysis.
JW and SJ supervised patient diagnosis and treatment. CT drafted the manuscript. JC and JW revised the manuscript. All authors critically reviewed the article and approved the final manuscript.

Funding This study has received funding by National Natural Science Foundation of China (81873917). The funder had no role in study design, data collection and analysis, decision to publish, or preparation of the manuscript.

Data availability The datasets used and analyzed during the current study are available from the corresponding author on reasonable request.

Code availability Not applicable.

\section{Compliance with ethical standards}

Conflict of interest All authors declare no conflicts of interest.

Ethical approval The study protocol was complied with the principles of the Declaration of Helsinki and was approved by the institutional review board of the Union Hospital, Tongji Medical college, Huazhong University of Science and Technology and Wuhan Jinyintan Hospital.

Consent to participate The institutional ethics committee of Wuhan Union Hospital and Wuhan Jinyintan Hospital agree to waive the consent to participate.

Consent for publication Not applicable.

\section{References}

Fahim A, Crooks MG, Wilmot R, Campbell AP, Morice AH, Hart SP (2012) Serum carcinoembryonic antigen correlates with severity of idiopathic pulmonary fibrosis. Respirology 17(8):1247-1252

Goldenberg DM, Neville AM, Carter AC, Go VLW, Hol-Yoke ED, Isselbacher KJ, Schwartz M (1981) Carcinoembryonic antigen: its role as a marker in the management of cancer. A National Institutes of Health Consensus Development Conference. Ann Internal Med 94(3):407-409

Guan WJ, Ni ZY, Hu Y, Liang WH, Ou CQ, He JX, Liu L, Shan H, Lei CL, Hui DSC et al (2020) Clinical characteristics of Coronavirus disease 2019 in China. N Engl J Med 18:1708-1720

Hoffmann M, Kleine-Weber H, Schroeder S, Kruger N, Herrler T, Erichsen S, Schiergens TS, Herrler G, Wu NH, Nitsche A et al (2020) SARS-CoV-2 cell entry depends on ACE2 and TMPRSS 2 and is blocked by a clinically proven protease inhibitor. Cell. https://doi.org/10.1016/j.cell.2020.02.052

Jin X, Lian JS, Hu JH, Gao J, Zheng L, Zhang YM, Hao SR, Jia HY, Cai H, Zhang XL et al (2020) Epidemiological, clinical and virological characteristics of 74 cases of coronavirus-infected disease 2019 (COVID-19) with gastrointestinal symptoms. Gut 6:1002-1009

Lin L, Jiang X, Zhang Z, Huang S, Zhang Z, Fang Z, Gu Z, Gao L, Shi H, Mai L et al (2020) Gastrointestinal symptoms of 95 cases with SARS-CoV-2 infection. Gut 69:997-1001 
Rule AH, Goleski-Reilly C, Sachar DB, Vandevoorde J, Janowitz HD (1973) Circulating carcinoembryonic antigen (CEA): relationship to clinical status of patients with inflammatory bowel disease. Gut 14(11):880-884

Xiao F, Tang M, Zheng X, Liu Y, Li X, Shan H (2020) Evidence for gastrointestinal infection of SARS-CoV-2. Gastroenterology 6:1831-1833
Publisher's Note Springer Nature remains neutral with regard to jurisdictional claims in published maps and institutional affiliations. 